\title{
Performance of the Front-End Electronics of the Qualification Model of DAMPE BGO Calorimeter in Environmental Tests and CERN Beam Experiment
}

\author{
Deliang Zhang ${ }^{1}$ \\ Department of Modern Physics, University of Science and Technology of China \\ Hefei, Anhui, 230026, China \\ State Key Laboratory of Particle Detection and Electronics(IHEP-USTC) \\ Hefei, Anhui, 230026, China \\ E-mail: dlzhangemail.ustc.edu.cn
}

\section{Changqing Feng*}

Department of Modern Physics, University of Science and Technology of China Hefei, Anhui, 230026, China

State Key Laboratory of Particle Detection and Electronics(IHEP-USTC)

Hefei, Anhui, 230026, China

E-mail: fengcqeustc.edu.cn

\section{Junbin Zhang}

Department of Modern Physics, University of Science and Technology of China Hefei, Anhui, 230026, China

State Key Laboratory of Particle Detection and Electronics(IHEP-USTC)

Hefei, Anhui, 230026, China

\section{Shanshan Gao}

Department of Modern Physics, University of Science and Technology of China Hefei, Anhui, 230026, China

State Key Laboratory of Particle Detection and Electronics(IHEP-USTC)

Hefei, Anhui, 230026, China

\section{Shubin Liu}

Department of Modern Physics, University of Science and Technology of China Hefei, Anhui, 230026, China

State Key Laboratory of Particle Detection and Electronics(IHEP-USTC)

Hefei, Anhui, 230026, China

\section{Qi An}

Department of Modern Physics, University of Science and Technology of China Hefei, Anhui, 230026, China

State Key Laboratory of Particle Detection and Electronics(IHEP-USTC)

Hefei, Anhui, 230026, China

\footnotetext{
${ }^{1}$ Speaker; *Corresponding Author

(c) Copyright owned by the author(s) under the terms of the Creative Commons Attribution-NonCommercial-ShareAlike Licence.
} 
The DAMPE (DArk Matter Particle Explorer) is a scientific satellite mainly aiming at indirectly searching for dark matter in space. One critical sub-detector of the DAMPE payload is an electromagnetic calorimeter, which consists of 308 BGO (Bismuth Germanate Oxid) crystal bars and 616 PMTs (photomultiplier tubes), for precisely measuring the energy of cosmic rays from $5 \mathrm{GeV}$ to $10 \mathrm{TeV}$. The $\mathrm{BGO}$ calorimeter, with 1848 readout channels and a dynamic range of $2 \times 10^{\wedge} 5$ for each crystal bar, is equipped with a complex readout system which contains 16 front-end electronics boards (FEE) with a total power consumption of $26 \mathrm{~W}$. The qualification model of the BGO calorimeter, as well as its front-end electronics, has been constructed and passed a series of environmental tests, such as EMC (Electromagnetic Compatibility) test, vibration test, thermal cycling test and thermal-vacuum test. The front-end electronics performed well and each electronics channel achieved a dynamic range of 0 to $12.5 \mathrm{pC}$ with a low noise better than $3 \mathrm{fC}$ and nonlinearity less than $2 \%$. Test results showed that it could adapt to the harsh space environments. From the fall of 2014, three accelerator beam tests were successfully carried out at CERN with PS and SPS facilities, which suggested that the design specifications of the BGO calorimeter and its front-end electronics were achieved.

The 34th International Cosmic Ray Conference

30 July- 6 August, 2015

The Hague, The Netherlands 


\section{Introduction}

The Dark Matter Particle Explorer (DAMPE) is a scientific satellite designed to fly in a near-earth orbit with an altitude of $500 \mathrm{~km}$ for a mission period of at least 3 years, searching for the clue of dark matter particles through obtaining the e+/e- and $\gamma$ energy spectrum in space. As shown in Fig. 1, the DAMPE payload consists of four sub-detectors from top to bottom: the Plastic Scintillation Detector (PSD), the Silicon-Tungsten Tracker (STK), the Bismuth Germanate Oxide (BGO) calorimeter, and the Neutron detector (ND) [1].

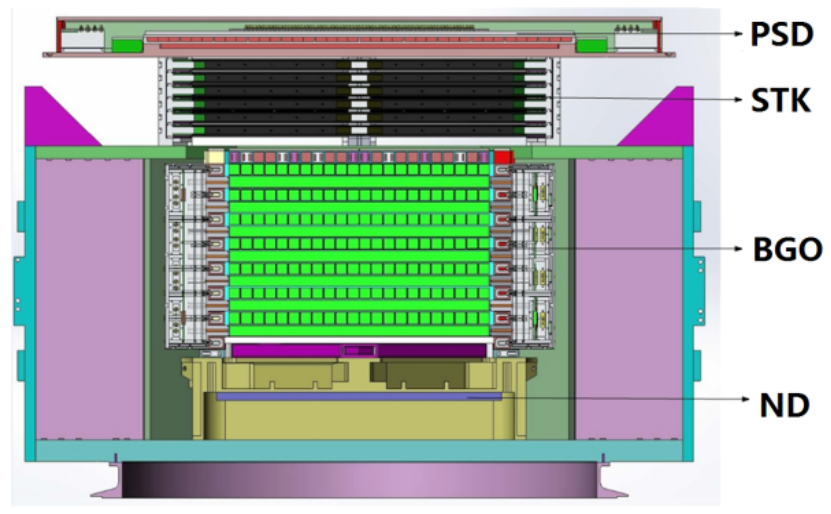

Fig. 1. Cross-section image of the DAMPE detector.

As a crucial sub-detector of the DAMPE payload, the BGO Calorimeter consists of 308 BGO (Bismuth Germanate Oxide) crystal bars, 616 PMTs (photomultiplier tubes) and 1848 dynode signals where two PMTs couple a crystal at both ends and each PMT outputs three dynode signals (dynode 2, dynode 5 and dynode 8). It is responsible for precisely measuring the energy of cosmic particles from $5 \mathrm{GeV}$ to $10 \mathrm{TeV}$, distinguishing positrons/electrons and gamma rays from hadron backgrounds, and providing trigger information for the whole DAMPE payload [2] [3].

The BGO detector's read-out electronics contains 16 front-end electronics boards (FEE) and 1848 electronics channels where each electronics channel deals with one dynode signal, measuring charge of the dynode signal.

The qualification model (QM) of the BGO calorimeter, as well as its front-end electronics, has been constructed and passed a series of tests such as functional tests, FEE board screening experiment, EMC (Electromagnetic Compatibility) test, vibration test, thermal cycling test, thermal-vacuum test and CERN beam test. The performance of the front-end electronics of QM BGO calorimeter is described in this paper.

\section{Front-End Electronics of the BGO Calorimeter}

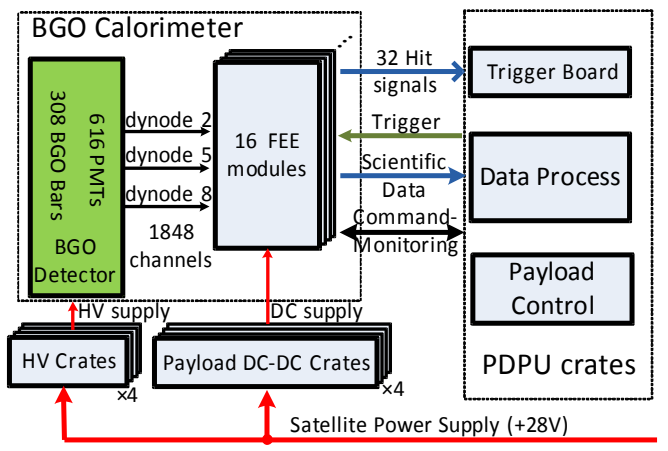

Fig. 2. Readout electronics for BGO calorimeter. 
Performance of the Front-End Electronics of the Qualification Model of DAMPE BGO Calorimeter in Environmental Tests and CERN Beam Experiment

Deliang Zhang, Changqing Feng et al

A read-out electronics systerm for BGO Calorimeter has been constructed to acquire the 1848 dynode signals. Fig. 2 shows the architecture of the readout electronics systerm for BGO calorimeter. It contains 16 front-end electronics boards (FEE) and 1848 electronics channels. The FEEs receive the current pulses from PMT dynodes, integrate the charges and digitize them under the control of trigger signals and then pack and transmit the measured results to PDPU.

Three types of FEE boards (FEE-A, FEE-B, FEE-C) are designed with the same schematic except for different numbers of channels and FEE-A's additional function of generating hit signals. The FEE boards are configed with the BGO crystal layers as Fig. 3. FEE-A and FEE-B contain 144 electronics channels which are responsible for acquiring dynode signals from two crystal layers and FEE-C contains 72 electronics channels which are only responsible for acquiring dynode signals from one crystal layer [3].

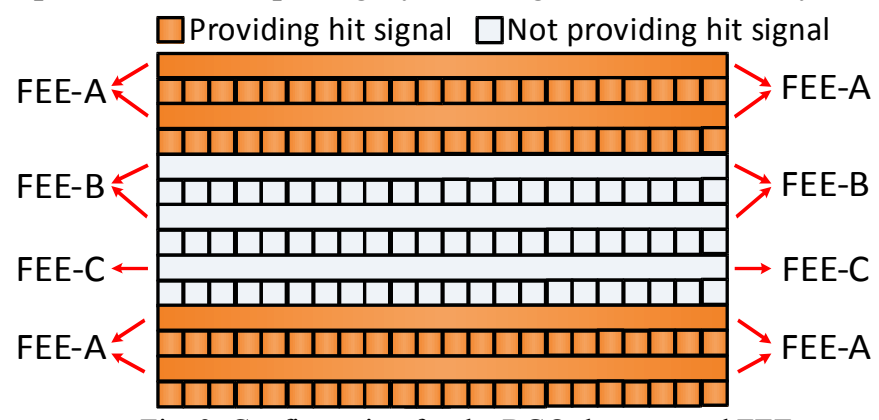

Fig. 3. Configuration for the BGO detector and FEEs.

We mainly introduce FEE-A in this paper. FEE-A's schematic is shown in Fig. 4. It contains several parts, charge measurement circuit, calibration circuit, hit signal generating circuit (only in FEE-A), current and temperature monitoring circuit, power supply circuit and communication circuit between FEE and the payload data process unit (PDPU), which are all controlled by an Actel Flash FPGA. Among them, the charge measurement circuit is heart of the FEE which is responsible for acquiring the detector's dynode signals. It's made up of 2 VA160s [6], 4 VATA160s [7], two operational amplifiers, a high-precision ADC (Analog to Digital Converter), a FPGA and user-defined serial data bus to PDPU. Fig. 5 shows the photograph of a FEE-A board [4] [5].

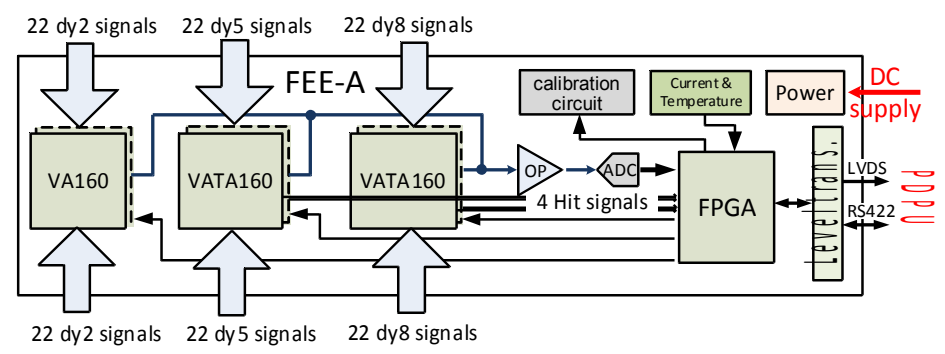

Fig. 4. The schematic of the FEE.

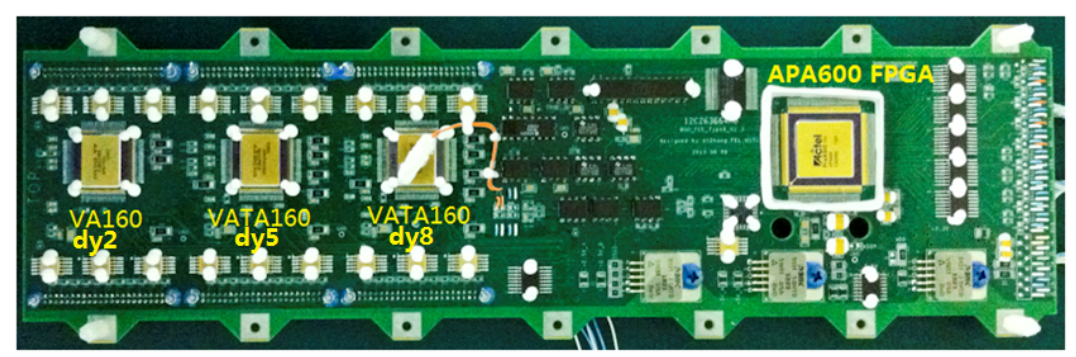

Fig. 5. Photograph of the FEE board (Type A) 
FEE-A contains 144 electronics channels. By receiving current pulse signals from PMT dynodes, integrating the charges in VA160s or VATA160s, amplifying through 2 level operational amplifiers and digitizing with the ADC, FEE acquires the charges of the input detector signals. The dynamic range should cover $0 \sim 12 \mathrm{pC}$, the precision should be better than $10 \mathrm{fC}$ and the nonlinearity should be less than $2 \%$ for each electronics channel [3].

\section{Functional Tests}

Some functional tests in room temperature have been done to confirm the the performance of the FEE boards. These tests are pedestal test, self calibration test and external signal test.

\subsection{Pedestal test}

Pedestal test results of a FEE-A are shown in Fig. 6, (a) is the pedestal average and (b) is the pedestal RMS of every channel. FEE-A contains 144 electronic channels. The pedestal test is carried out with the FEE shielded in a metal box and its input ports floating. The pedestals vary between -250 to 250 ADC code, which can be deducted during scientific data processing. The RMS is lower than 2.4 ADC code (equivalent to $2.4 \mathrm{fC}$ ), which means the FEE achieves a low noise (lower than $3 \mathrm{fC}$ ).

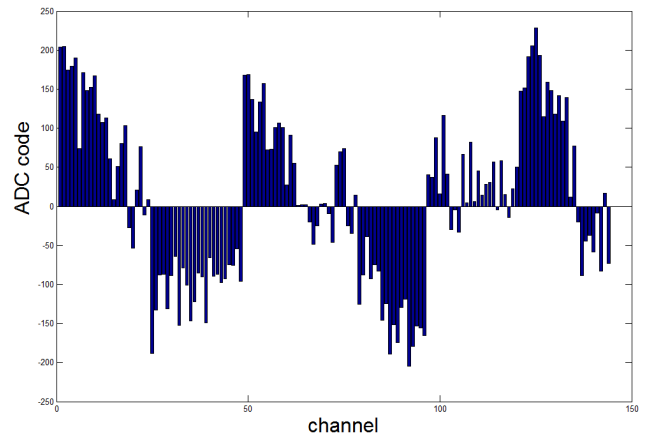

(a)

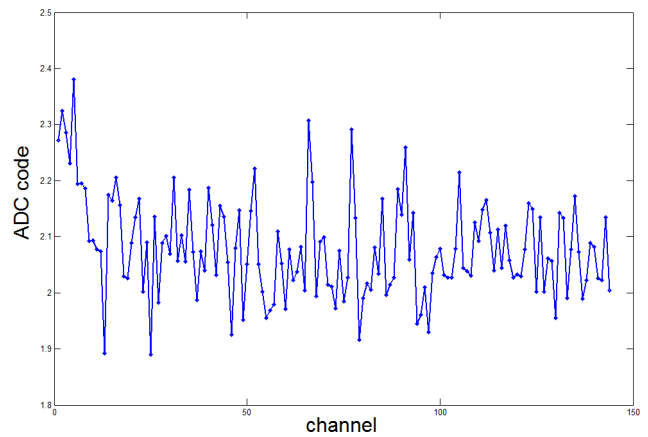

(b)

Fig. 6. Pedestal test results of all channels on a FEE. (a) is average of the pedestal, (b) is RMS of the pedestal

\subsection{Calibration test}

Calibration test is carried out with the calibration circuit on FEE. The calibration circuit on FEE is designed to perform onboard self-test. Fig. 7 shows the calibration result of a channel on FEE. The x-axis is the input calibration signal charge and the $y$-axis is the output measured ADC code. The result tells us that the FEE achieves a good linearity and a dynamic range of $0 \sim 14 \mathrm{pC}$.

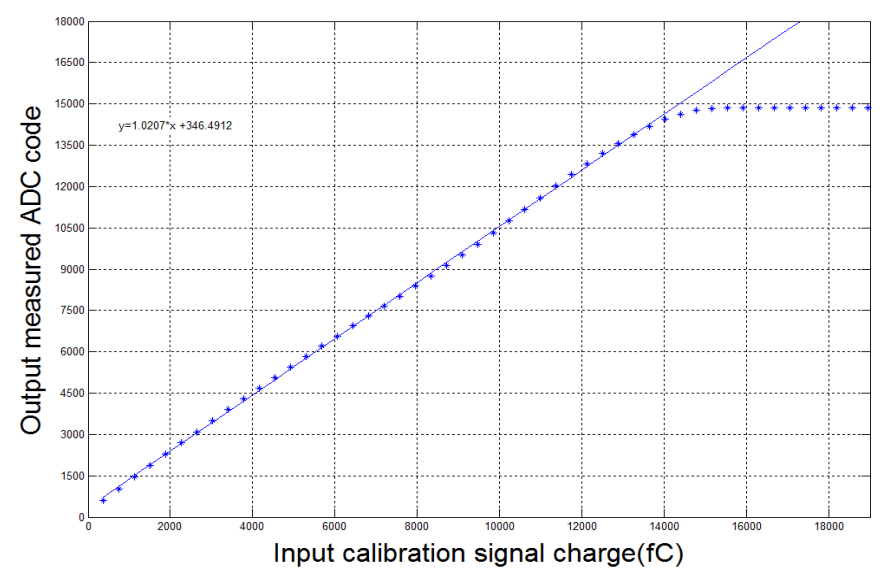

Fig. 7. Calibration result of a channel on FEE 


\subsection{External signal test}

External signal test is carried out with a function generator (Tek AFG3252) to simulate acquiring scientific data. Fig. 8 shows the external signal test result of a channel on FEE. The $x$-axis is the input signal charge and the $\mathrm{y}$-axis is the output measured ADC code. The external signal test result is similar with the calibration result. FEE achieves a good linearity (nonlinearity is small than $2 \%$ ) and a dynamic range of $0 \sim$ $12.5 \mathrm{pC}$ for each electronics channel.

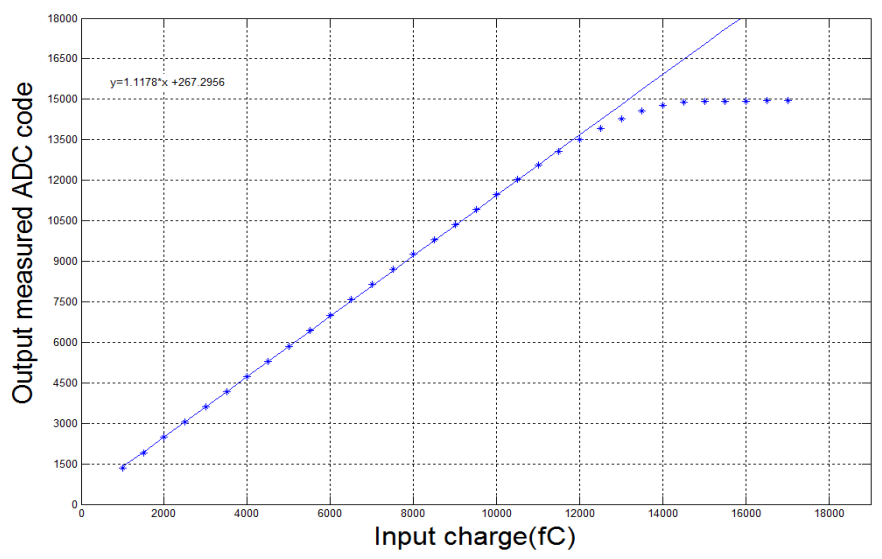

Fig. 8. External signal test result of a channel on FEE

\section{4. $\quad$ FEE board screening experiment}

FEE board screening experiment is temperature cycling test in atmospheric pressure for aging the components on FEE. Temperature cycling test consists of 16.5 cycles. For the first cycle and the last two cycles, each consists of 0.5 hour in $75^{\circ} \mathrm{C}, 2$ hours in $55^{\circ} \mathrm{C}, 0.5$ hour in $-45^{\circ} \mathrm{C}$ and 2.5 hours in $-25^{\circ} \mathrm{C}$. For the other 13.5 cycles, each consists of only 1.5 hours in $75^{\circ} \mathrm{C}$ and 2 hours in $-45^{\circ} \mathrm{C}$. The FEE is powered on only in $-25^{\circ} \mathrm{C}, 25^{\circ} \mathrm{C}$ and $55^{\circ} \mathrm{C}$ during the first cycle and the last two cycles. We learn about the performance of the FEE through onboard calibration, pedestal and remote state monitoring when FEE is powered on.

Fig. 9 shows the calibration results in different temperature. The gain coefficient of the electronic channel differs in different temperature where it decreases with temperature rising. So we can compensate the measured scientific data with onboard calibration results in different temperature.

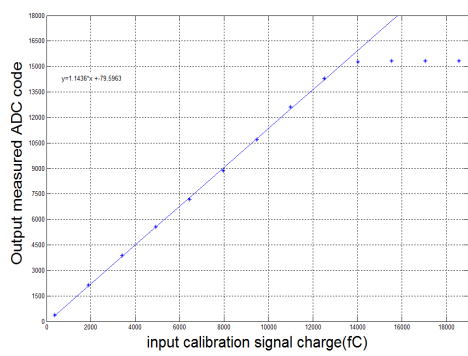

(a) $-25^{\circ} \mathrm{C}$

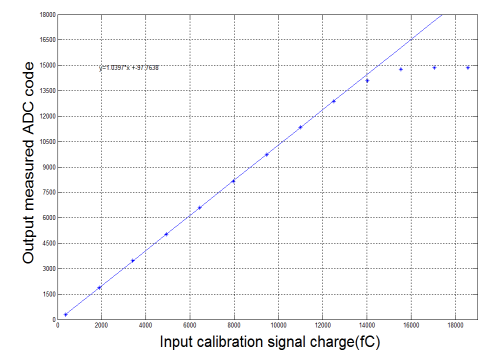

(b) $25^{\circ} \mathrm{C}$

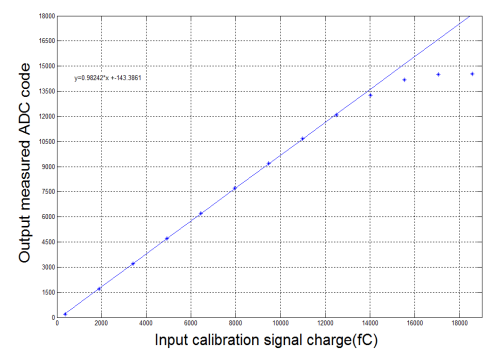

(c) $55^{\circ} \mathrm{C}$

Fig.9. Calibration results of a channel on FEE during temperature cycling test. (a) the gain coefficient in $-25^{\circ} \mathrm{C}$ is 1.14 , (b) the gain coefficient in $25^{\circ} \mathrm{C}$ is 1.04 , (c) the gain coefficient in $55^{\circ} \mathrm{C}$ is 0.98 . 
Performance of the Front-End Electronics of the Qualification Model of DAMPE BGO Calorimeter in Environmental Tests and CERN Beam Experiment

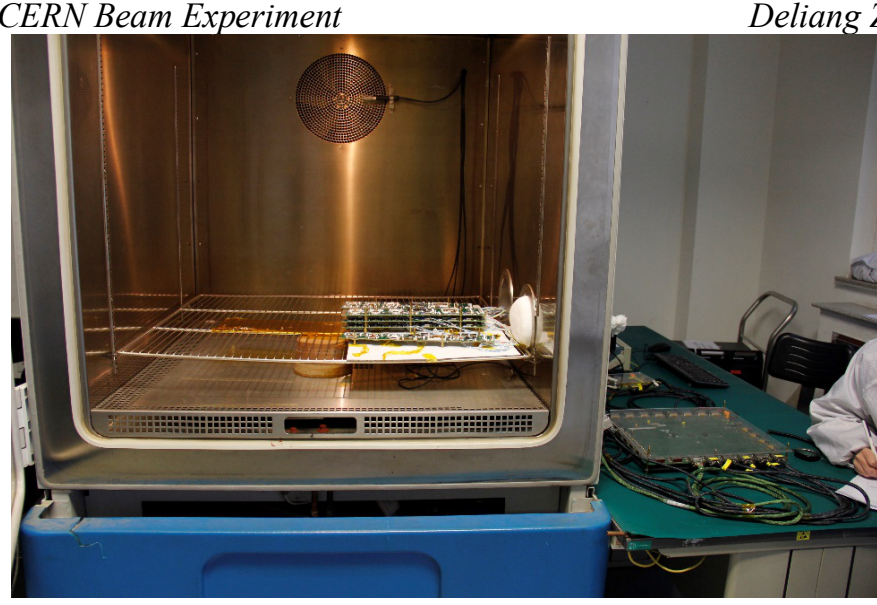

Fig.10. Experiment site of temperature cycling test.

\section{Environmental Tests and CERN Beam Test}

The QM BGO calorimeter has gone through a series of environmental tests, including the EMC test, vibration test, thermal cycling test, thermal-vacuum test. During these tests, the front-end electronics performed well and achieved a aerospace grade reliability.

From last fall, three beam tests have been successfully conducted at CERN with PS and SPS facilities. The beam tests included electron beam with energy range of $0.5 \sim 243 \mathrm{GeV}$, proton beam with energy range of $10 \sim 400 \mathrm{GeV}$ and heavy ion beam with energy range of $40 \sim 75 \mathrm{GeV}$. The beam test results confirmed the good performances of the front-end electronics in QM BGO Calorimeter, such as the energy linearity and resolution, which satisfies the physics requirements.

Fig. 10 shows the sites of some environmental tests and CERN beam test.

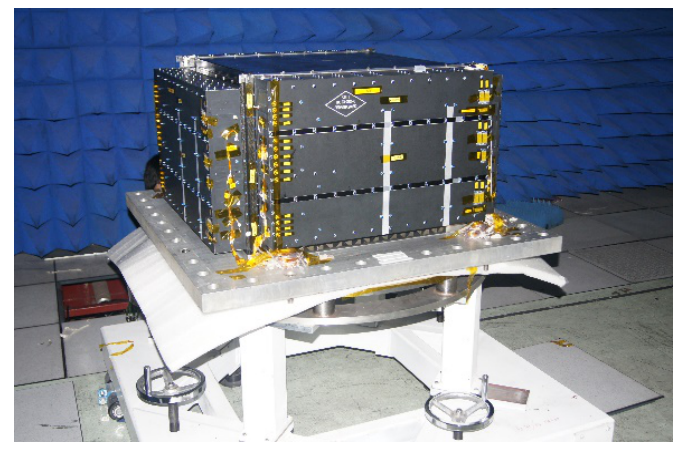

(a) EMC test

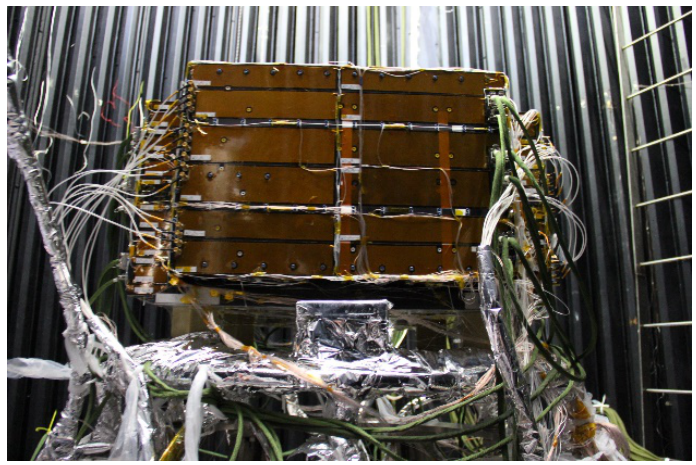

(c) thermal-vacuum test

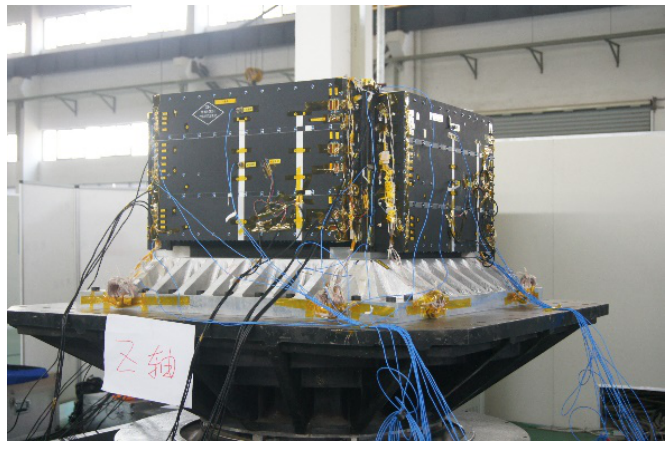

(b) vibration test

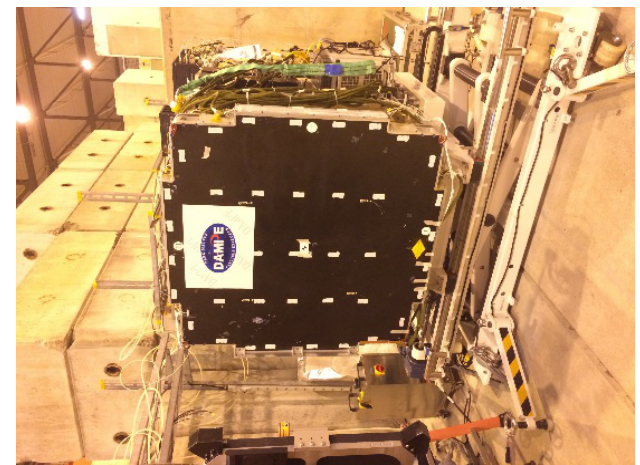

(d) CERN beam test

Fig.11. Photographs of some environmental tests and CERN beam test sites. 


\section{Conclusion}

The qualification model of the BGO calorimeter, as well as its front-end electronics, has been constructed and passed a series of tests including functional tests, FEE board screening experiment, EMC test, vibration test, thermal cycling test, thermal-vacuum test and CERN beam tests. The front-end electronics system performed well and each electronics channel achieved a dynamic range of 0 to $12.5 \mathrm{pC}$ with a resolution better than $3 \mathrm{fC}$ and nonlinearity less than $2 \%$.

Test results showed that the front-end electronics achieved a high reliability which can adapt to the harsh space environment and a good performance which can satisfy the physics requirements.

\section{Acknowledgment}

We would like to thank Dr. Yunlong Zhang, Dr.Zhiyong Zhang and Mr. Sicheng Wen for helpful discussions and useful suggestions. We also would like to thank all the people from DAMPE collaboration who helped make this work possible.

This work was supported by the Strategic Priority Research Program on Space Science of Chinese Academy of Sciences (Grant No. XDA04040202-4), and the National Basic Research Program (973 Program) of China (Grant No. 2010CB833002).

\section{References}

[1] Yunlong Zhang, The Study of an EM Calorimeter for Searching Dark Matter in Space, University of Science and Technology of China, 2011.

[2] Changqing Feng, The Research on the Readout Electronics for the Calorimeter Detector of the Dark Matter Exploration Satellite, University of Science and Technology of China, 2011.

[3] Changqing Feng, Deliang Zhang, et al., Design of the Readout Electronics for the Qualification Model of DAMPE BGO Calorimeter, Presented at 19th IEEE Real-Time Conference, May 2014, Nara, Japan, Available: http://arxiv.org/ftp/arxiv/papers/1406/1406.3886.pdf.

[4] Shanshan Gao, Changqing Feng, Yunlong Zhang, et al., The BGO Electromagnetic Calorimeter Sub-trigger Electronics for DAMPE, presented at 19th IEEE Real-Time Conference, Nara, Japan, May 2014, Available: http://ieeexplore.ieee.org/xpl/articleDetails.jsp?reload=true\&arnumber=7097422

[5] Di Jiang, Changqing Feng, et al., Design of a Test Platform for Screening Procedures of VA160 and VATA160 ASICs in DAMPE Mission, Presented at 19th IEEE Real-Time Conference, May 2014, Nara, Japan, Available: http://inspirehep.net/record/1367408

[6] VA160 datasheet, Integrated Detector Electronics AS (IDEAS), Nydalen, Oslo, Norway, 2013. http://www.ideas.no.

[7] VATA160 datasheet, Integrated Detector Electronics AS (IDEAS), Nydalen, Oslo, Norway, 2013. http://www.ideas.no. 